\title{
COMORBILIDAD ENTRE ABUSO/DEPENDENCIA DE DROGAS Y EL DISTRÉS PSICOLÓGICO EN SIETE PAÍSES DE LATINOAMÉRICA Y UNO DEL CARIBE
}

\author{
Edgar Merchán-Hamann ${ }^{1}$,Erotildes Maria Leal2, Liliana Basso Musso ${ }^{3}$, Miriam García Estrada ${ }^{4}$, Patrice \\ Reid ${ }^{5}$, Olga Vladimirovna Kulakova ${ }^{6}$, Eddy Vásquez Espinoza ${ }^{7}$, Opal Jones Willis ${ }^{8}$, Ricardo Prieto López ${ }^{9}$, \\ Diana Domenech ${ }^{10}$
}

\author{
${ }^{1}$ Ph.D. Universidad de Brasília, Brasil. E-mail: merchan.hamann@gmail.com \\ 2 Ph.D. Universidad Federal de Rio de Janeiro, Brasil. E-mail: eroleal@gmail.com \\ ${ }^{3}$ Ph.D. Universidad de Valparaíso, Chile. E-mail: liliana.basso@uv.cl \\ ${ }^{4}$ MSc. Universidad de San Carlos, Guatemala. E-mail: andragogiamiriam@yahoo.com \\ ${ }^{5}$ MSc. University of the West Indies - Mona Campus, Jamaica. E-mail: patrice.a.reid@gmail.com \\ ${ }^{6}$ MSc. Universidad Nacional Autónoma de Nicaragua. León, Nicaragua. E-mail: olgvik20@yahoo.es \\ ${ }^{7}$ MSc. Universidad Nacional Autonoma de Nicaragua. León, Nicaragua. E-mail: eddyarnulfo@gmail.com \\ ${ }^{8}$ MSc. Universidad de Panamá, Panamá. E-mail: opaljones24@hotmail.com \\ ${ }_{9}^{9}$ MSc. Universidad Iberoamericana, Paraguay. E-mail: ricardoprilo@yahoo.com \\ ${ }^{10}$ MSc. Universidad de la República, Uruguay. E-mail: ddomenech@fmed.edu.uy
}

\begin{abstract}
RESUMEN: Fue realizado estudio multicéntrico en pacientes bajo tratamento por dependencia de alcohol y substancias ilícitas, en ocho países (Brasil, Chile, Guatemala, Jamaica, Nicaragua, Panamá, Paraguay, Uruguay). El objetivo fue investigar la frecuencia de distrés psicológico, diagnóstico actual de comorbilidades, y disfuncionalidad familiar percibida. Fueron incluídos 1.073 voluntarios adultos, que diligenciaron un cuestionario o fueron entrevistados. El distrés psicológico y la disfuncionalidad familiar fueron evaluados por escalas (Kessler K-10 y APGAR-family). Hombres predominaron en todos los lugares (edades entre 18 y 86). En la mayoría de los sitios, el diagnóstico actual de ansiedad varió entre 30\% y 40\% y el de depresión entre $20 \%$ y $35 \%$. Niveles altos u muy altos de distrés psícológico fueron reportados por más de 70\% en Uruguay, Nicaragua, Guatemala y Brasil. La disfuncionalidad familiar severa fue mayor en Panamá $34.7 \%$ seguida de los sitios de Nicaragua 20-25\%. La prevalencia de distrés psicológico sugiere niveles altos de comorbilidad.
\end{abstract}

DESCRIPTORES: Comorbilidad. Distrés psicológico. Trastornos relacionados con sustancias. Atención en salud mental.

\section{COMORBIDITY OF DRUG ABUSE/DEPENDENCE AND PSYCHOLOGICAL DISTRESS IN SEVEN COUNTRIES OF LATIN AMERICA AND ONE OF THE CARIBBEAN}

\begin{abstract}
A multicenter study among patients in treatment for alcohol and illicit drugs abuse were conducted in eight countries (Brazil, Chile, Guatemala, Jamaica, Nicaragua, Panama, Paraguay, Uruguay). Our objective was to ascertain the frequency of psychological distress, current diagnosis of comorbidities and perceived familiar dysfunction. It was recruited 1,073 adult volunteers and they filled out a questionnaire or were interviewed. Psychological distress was evaluated through the Kessler's K-10 scale and family dysfunction by the APGAR-family scale. Male individuals predominated at all study sites (age range: 18-86). Current diagnosis of anxiety ranged from 30.0\% to $40.0 \%$ in most sites. Current diagnosis of depression ranged from $20 \%$ to $35 \%$ in most sites. High and very high levels of psychological stress were higher than $70 \%$ in Uruguay, Nicaragua, Guatemala, and Brazil. Severe family dysfunction was higher in Panamá 34.7\% followed by Nicaraguan cities 20-25\%. The prevalence of psychological distress suggests higher rates of comorbidity.
\end{abstract}

DESCRIPTORS: Comorbidity. Psychological distress. Substance use-related disorders. Mental health assistance.

\section{COMORBIDADE ENTRE ABUSO/DEPENDENNCIA DE DROGAS E O SOFRIMENTO PSÍQUICO EM SETE PAÍSES DA AMÉRICA LATINA E UM DO CARIBE}

\begin{abstract}
RESUMO: Foi realizado um estudo multicéntrico em pacientes sob tratamento para dependência de álcool e substâncias ilícitas em oito países (Brasil, Chile, Guatemala, Jamaica, Nicarágua, Panamá, Paraguai, Uruguai). O objetivo foi averiguar a frequência de sofrimento psíquico, diagnóstico atual de comorbidades, e disfuncionalidade familiar percebida. Foram incluídos 1.073 voluntários adultos, que preencheram um questionário ou foram entrevistados. O sofrimento psíquico e a disfuncionalidade familiar foram avaliados por escalas (Kessler K-10 e APGAR-family). Os homens predominaram em todos os locais (idades entre 18 e 86). Na maioria dos locais, o diagnóstico atual de ansiedade variou de $30 \%$ a $40 \%$ e o de depressão, de $20 \%$ a 35\%. Níveis altos e muito altos de sofrimento psíquico foram relatados por mais de 70\% em Uruguai, Nicarágua, Guatemala e Brasil. Disfuncionalidade familiar severa foi maior no Panamá $34,7 \%$, seguida da Nicarágua 20-25\%. A prevalencia de sofrimento psíquico sugere níveis mais altos de comorbidade.
\end{abstract}

DESCRITORES: Comorbidade. Sofrimento psíquico. Transtornos relacionados ao uso de substancias. Assistência em saúde mental.

Texto Contexto Enferm, Florianópolis, 2012; 21 (Esp): 87-95. 


\section{INTRODUCCIÓN}

La comorbilidad entre abuso/dependencia de substancias psicotrópicas y otros trastornos mentales, a pesar de conocida desde hace mucho tiempo, ha sido ampliamente estudiada desde la década de 1990. La dependencia constituye un diagnóstico de trastorno mental que puede coexistir con otros disturbios psiquiátricos, lo cual se transforma en un desafío para servicios de atención a la salud y sus respectivos profesionales. Por eso es relevante determinar la frecuencia de la comorbilidad y hasta qué punto los centros de tratamiento están diagnosticando y tratando estos eventos.

Varios estudios han abordado estos problemas ${ }^{1}$ revelando una prevalencia de comorbilidades variable entre los pacientes que se encuentran en tratamiento para dependencia de alcohol y drogas $(\sim 50 \%)$ tienen un diagnóstico psiquiátrico: $26 \%$ tienen trastornos de ánimo/humor, 28\% de ansiedad, $18 \%$ de personalidad antisocial y $7 \%$ esquizofrenia. Datos similares también fueron hallados en publicaciones más recientes en todo el mundo..$^{2-3}$ Numerosos estudios epidemiológicos se han realizado en los últimos 15 años y han demostrado que muchos trastornos psiquiátricos y trastornos por consumo de sustancias co-ocurren con más frecuencia de lo que cabría esperar. ${ }^{4}$

Una manera de acceder de manera rápida a los diagnósticos más prevalentes es la aplicación de instrumentos de rastreo para determinar el grado de distrés psicológico (en portugués, sofrimento psíquico). ${ }^{5}$

Además de la comorbilidad, un tema abordado con frecuencia que incide en la recuperación y reintegración social de personas con dependencia, es el ambiente familiar. A partir de investigaciones realizadas en las décadas pasadas, fue evidente a asociación de percepción de disfunción familiar con la dependencia. ${ }^{6}$ En este sentido, la aplicación de escalas simples para mensurar la funcionalidad familiar ha sido un recurso frecuentemente utilizado. ${ }^{7}$ Pocos estudios han sido realizados sobre comorbilidad en Latinoamérica y el Caribe. ${ }^{8}$

El objetivo de esta investigación fue averiguar la prevalencia del distrés psicológico en los pacientes que están recibiendo atención en centros especializados de ocho países de la región de las Américas. Este artigo destaca aspectos descriptivos relacionados con variables socio-demográficas, diagnósticos actuales de comorbilidades más frecuentes (ansiedad y depresión), el distrés psicológico y la disfuncionalidad familiar .

\section{MÉTODO}

Se realizó un estudio epidemiológico descriptivo de prevalencia, dirigido a la población adulta que está en tratamiento por abuso/dependencia de drogas en centros de tratamiento especializado de Brasil, Chile, Guatemala, Jamaica, Nicaragua, Panamá, Paraguay, Uruguay. La investigación fue realizada entre 2009 y 2010, siendo invitados a participar de la misma, pacientes con 18 años o más que voluntariamente proporcionaran su consentimiento.

Fueron excluidos de participación, pacientes incapaces de contestar el cuestionario debido a deficiencia mental, intoxicación, y/o quienes se negaran a dar su consentimiento informado. Estudiantes de pregrado, postgrado y profesionales de salud entrenados hicieron parte del equipo de recolección de los datos y, ocasionalmente, los coordinadores. Los pacientes diligenciaron un cuestionario auto-administrado. Miembros del equipo de recolección de datos ayudaron a los participantes en caso de dudas o, cuando los voluntarios no sabían leer o escribir, llevaron a cabo entrevistas.

Los datos se recogieron por medio del formulario EULAC-CICAD, que fue concebido como un instrumento de admisión a servicios de salud y posteriormente adaptado para este estudio con el debido permiso. El EULAC-CICAD formulario consta de 30 preguntas sobre características sociodemográficas, historia de uso/abuso de drogas y alcohol, trastornos mentales diagnosticados (tanto pasados como actuales), y aspectos del cuidado recibido en los centros de tratamiento.

El distrés psicológico fue evaluado por medio del Instrumento Kessler-10 (K-10), que documenta diez preguntas sobre la vida en el último mes, siendo que los valores atribuidos por los pacientes es sumada. ${ }^{5}$ Los valores sumados pueden variar de 10 (que indica ausencia o niveles mínimos de distrés psicológico) a 50 (que indica niveles altos). Las puntuaciones obtenidas han llevado a la clasificación del distrés en cuatro categorías: bajo (10-15), moderado (16-21), severo o alto (22-29) y muy severo o muy alto (30-50). Para efectos de análisis, las dos últimas categorías son unidas (distrés severo y muy severo). La escala es más sensible para ansiedad o depresión. ${ }^{9}$

La disfuncionalidad familiar fue evaluada por la escala APGAR-familiar ${ }^{7}$. Esta escala contiene cinco dimensiones (la adaptabilidad, asociación, crecimiento, afecto y resolución de conflictos), 
que documentan la satisfacción percibida por los participantes con respecto a las relaciones dentro de su familia. Cada dimensión puede tener valores de cero, un o dos y la suma total puede alcanzar un máximo de 10 puntos. Una puntuación total de siete o más sugiere una percepción de alta funcionalidad familiar; entre cuatro y seis, sugiere una moderada disfunción familiar; y de tres o menos, una severa disfunción familiar.

Fue utilizado el software Epi Info versión 3.5.1 desarrollado por el CDC, para introducir y analizar los datos. Para el análisis preliminar de datos fueron calculadas las proporciones de variables dicotómicas y otras categóricas (prevalencias). Para las variables numéricas fueron calculadas las medidas de tendencia central y de dispersión.

El estudio fue sometido al Research Ethics Board del Centro de Adicción y Salud Mental (REBCAMH), afiliado a la Universidad de Toronto, y al Comité de Ética en Investigación de las respectivas instituciones de los países participantes siendo aprobado en todos ellos.

\section{RESULTADOS}

En total de 1.073 personas participaran del estudio, siendo que en todos los lugares predominaron los hombres, variando de $64.5 \%$ en Valparaíso - Chile a $97.6 \%$ en León - Nicaragua (Tabla 1). La edad varió entre de 18 a 86 años siendo que en algunos lugares predominaron sujetos más jóvenes, como en Montevideo, en donde $100 \%$ son menores de 40 años, comparado con Brasilia - Brasil, en donde ese porcentaje corresponde a $38.8 \%$. El nivel de escolaridad también fue variable. Hubo más personas analfabetas y de baja escolaridad en Brasilia - Brasil 41.2\%, comparado con países en que ese nivel educacional era más raro, como fue el caso de Jamaica $1.8 \%$ o Paraguay $4.7 \%$. En contraste, la mayor proporción de pacientes con educación universitaria (completa o incompleta) se encontraba en Parroquias de Jamaica 39.3\% y de Valparaíso Chile $32.0 \%$, porcentajes altos comparados con los encontrados en Montevideo - Uruguay $9.8 \%$ y en las provincias de Panamá $11.0 \%$.

Tabla 1 - Distribución por sexo, edad y niveles educacionales entre pacientes en los centros de tratamiento para abuso y dependencia de drogas según ciudades y países 2009/2010

\begin{tabular}{|c|c|c|c|c|c|c|c|c|c|c|c|c|c|c|c|c|c|c|c|c|c|c|}
\hline \multirow[t]{3}{*}{ Ciudades/ Países } & \multicolumn{4}{|c|}{ Sexo } & \multicolumn{4}{|c|}{ Edad } & \multicolumn{14}{|c|}{ Nível educacional* } \\
\hline & \multicolumn{2}{|c|}{ Masc. } & \multicolumn{2}{|c|}{ Fem. } & \multicolumn{2}{|c|}{$>=40$} & \multicolumn{2}{|c|}{$<40$} & \multicolumn{2}{|c|}{$\begin{array}{c}\text { Sin } \\
\text { escuela }\end{array}$} & \multicolumn{2}{|c|}{$\begin{array}{c}1^{\circ} \\
\text { incompl. }\end{array}$} & \multicolumn{2}{|c|}{$\begin{array}{c}1^{\circ} \\
\text { completo }\end{array}$} & \multicolumn{2}{|c|}{$\begin{array}{c}2^{\circ} \\
\text { incompl. }\end{array}$} & \multicolumn{2}{|c|}{$\begin{array}{c}2^{\circ} \\
\text { completo }\end{array}$} & \multicolumn{2}{|c|}{$\begin{array}{c}3^{\circ} \\
\text { incompl. }\end{array}$} & \multicolumn{2}{|c|}{$\begin{array}{c}3^{\circ} \\
\text { completo }\end{array}$} \\
\hline & $\mathbf{n}$ & $\%$ & $\mathrm{n}$ & $\%$ & $\mathbf{N}$ & $\%$ & $\mathbf{n}$ & $\%$ & $\mathbf{n}$ & $\%^{\dagger}$ & $\mathbf{n}$ & $\%$ & $\mathrm{n}$ & $\%$ & $\mathrm{n}$ & $\%$ & $\mathbf{n}$ & $\%$ & $\mathbf{n}$ & $\%$ & $\mathbf{n}$ & $\%$ \\
\hline Brasília/ Brasil & 148 & 89.7 & 17 & 10.3 & 101 & 61.2 & 64 & 38.8 & 8 & 4.8 & 60 & 36.4 & 17 & 10.3 & 17 & 10.3 & 40 & 24.2 & 17 & 10.3 & 6 & 3.6 \\
\hline Macaé/ Brasil & 53 & 88.3 & 7 & 11.7 & 24 & 40.3 & 36 & 59.7 & & - & 18 & 30.0 & 9 & 15.0 & 6 & 10.0 & 18 & 30.0 & 3 & 5.0 & 6 & 10.0 \\
\hline Valparaíso/ Chile & 69 & 64.5 & 38 & 35.5 & 29 & 27.6 & 76 & 72.4 & 2 & 1.8 & 11 & 11.3 & 5 & 4.7 & 19 & 17.9 & 34 & 32.0 & 17 & 16.0 & 17 & 16.0 \\
\hline $\begin{array}{l}\text { Ciudad Guatemala/ } \\
\text { Guatemala }\end{array}$ & 87 & 95.6 & 4 & 4.4 & 29 & 31.9 & 61 & 67.4 & & - & 13 & 14.3 & 14 & 15.4 & 21 & 23.1 & 25 & 27.5 & 13 & 14.3 & 4 & 4.4 \\
\hline $\begin{array}{l}\text { Parroquias/ } \\
\text { Jamaica }\end{array}$ & 48 & 85.7 & 8 & 14.3 & 30 & 53.6 & 26 & 46.4 & & - & 1 & 1.8 & 2 & 3.6 & 11 & 19.6 & 20 & 35.7 & 12 & 21.4 & 10 & 17.9 \\
\hline León/ Nicaragua & 40 & 97.6 & 1 & 2.4 & 13 & 31.5 & 28 & 68.5 & 1 & 2.4 & 13 & 31.7 & 4 & 9.8 & 14 & 34.1 & 3 & 7.3 & 2 & 4.9 & 3 & 7.3 \\
\hline $\begin{array}{l}\text { Distrito Managua/ } \\
\text { Nicaragua }\end{array}$ & 102 & 79.7 & 26 & 20.3 & 38 & 29.3 & 92 & 70.7 & 6 & 4.7 & 19 & 14.7 & 24 & 18.6 & 38 & 29.5 & 19 & 14.7 & 10 & 7.8 & 13 & 10.1 \\
\hline Provincias/ Panamá & 133 & 79.6 & 34 & 20.4 & 109 & 65.4 & 58 & 34.6 & 5 & 3.0 & 14 & 8.4 & 19 & 11.4 & 74 & 44.3 & 35 & 21.0 & 13 & 7.8 & 7 & 4.2 \\
\hline Asunción/ Paraguay & 132 & 88.6 & 17 & 11.4 & 36 & 24.6 & 113 & 75.4 & 3 & 2.0 & 4 & 2.7 & 14 & 9.4 & 50 & 33.6 & 44 & 29.5 & 27 & 18.1 & 7 & 4.7 \\
\hline $\begin{array}{l}\text { Montevideo/ } \\
\text { Uruguay }\end{array}$ & 112 & 84.2 & 21 & 15.8 & & - & 134 & 100 & & - & 11 & 8.3 & 23 & 17.3 & 76 & 57.1 & 10 & 7.5 & 5 & 3.8 & 8 & 6.0 \\
\hline
\end{tabular}

${ }^{*} 1^{\circ}$ Escuela primaria o fundamental; $2^{\circ}$ Escuela secundaria o educación media; $3^{\circ}$ enseñanza universitaria; ${ }^{\dagger}$ Algunos porcentajes no contabilizan $100 \%$ debido a datos faltantes (missing data).

La tabla 2 contiene datos relacionados con la primera substancia mencionada como la más relevante para los pacientes. La bebida alcohólica fue relatada por $70 \%$ de los pacientes en Managua - Nicaragua y Brasilia - Brasil, seguido de Macaé Brasil $53.3 \%$ y Guatemala $52.8 \%$. La segunda droga más mencionada fue el crack (piedra de cocaína con otras substancias), relatada por cerca de $44 \%$ de los pacientes de León - Nicaragua, seguida por Jamaica $41.1 \%$ y Guatemala 21.3\%. La marijuana fue mencionada por $34.7 \%$ y $32.2 \%$ de los pacientes de centros de tratamiento de Panamá y Asunción - Paraguay, respectivamente.

La cocaína en polvo fue relatada por cerca de una cuarta parte de los pacientes de Valparaíso - Chile, Macaé - Brasil, Jamaica y Panamá. La pasta base de cocaína fue la droga en que hubo mayores disparidades. Ella fue mencionada como substancia más relevante por $71.6 \%$ de los pacientes de Montevideo - Uruguay, seguida por los de 
Valparaíso - Chile $40.2 \%$ y por solo $4.8 \%$ de los de paciente de los siete sitios restantes se refirieron Brasilia - Brasil. Vale la pena destacar que ningún a esta droga.

Tabla 2 - Droga principal por la cual se buscó atención y forma de uso de los pacientes en los centros de tratamiento para abuso y dependencia de drogas, según ciudades y países, 2009/2010

\begin{tabular}{|c|c|c|c|c|c|c|c|c|c|c|}
\hline \multirow{3}{*}{ Ciudades/Países } & \multicolumn{10}{|c|}{ Primera droga mencionada } \\
\hline & \multicolumn{2}{|c|}{ Alcohol } & \multicolumn{2}{|c|}{ Marijuana } & \multicolumn{2}{|c|}{ Crack } & \multicolumn{2}{|c|}{ Pasta base } & \multicolumn{2}{|c|}{ Cocaína } \\
\hline & $\mathbf{n}$ & $\%$ & $\mathbf{n}$ & $\%$ & $\mathbf{n}$ & $\%$ & $\mathbf{n}$ & $\%$ & $\mathbf{n}$ & $\%$ \\
\hline $\begin{array}{l}\text { Brasília/Brasil } \\
n=65\end{array}$ & 114 & 69.1 & 11 & 6.7 & 25 & 15.2 & 8 & 4.8 & 7 & 4.2 \\
\hline $\begin{array}{l}\text { Macaé/Brasil } \\
\mathrm{n}=60\end{array}$ & 32 & 53.3 & 3 & 5.0 & 9 & 15.0 & - & - & 15 & 25.0 \\
\hline $\begin{array}{l}\text { Valparaíso/Chile } \\
n=107\end{array}$ & 25 & 23.4 & 6 & 5.6 & - & - & 43 & 40.2 & 27 & 25.2 \\
\hline $\begin{array}{l}\text { Parroquias/Jamaica } \\
n=56\end{array}$ & 9 & 16.1 & 18 & 32.1 & 18 & 32.1 & - & - & 10 & 17.9 \\
\hline $\begin{array}{l}\text { Guatemala } \\
n=91\end{array}$ & 47 & 52.8 & 12 & 13.5 & 19 & 21.3 & - & - & 8 & 9.0 \\
\hline $\begin{array}{l}\text { León/Nicarágua } \\
n=41\end{array}$ & 15 & 36.6 & 7 & 17.1 & 18 & 43.9 & - & - & - & - \\
\hline $\begin{array}{l}\text { Managua/Nicarágua } \\
n=130\end{array}$ & 92 & 70.8 & 16 & 12.3 & 15 & 11.5 & - & - & 7 & 5.4 \\
\hline $\begin{array}{l}\text { Panamá } \\
\mathrm{n}=167\end{array}$ & 40 & 24.0 & 58 & 34.7 & 24 & 14.4 & - & - & 40 & 24.0 \\
\hline $\begin{array}{l}\text { Asunción/Paraguai } \\
n=149\end{array}$ & 59 & 39.6 & 48 & 32.2 & 24 & 16.1 & - & - & 17 & 11.4 \\
\hline $\begin{array}{l}\text { Montevideo/Uruguai. } \\
n=34\end{array}$ & 4 & 3.0 & 9 & 6.7 & 3 & 2.2 & 96 & 71.6 & 21 & 15.7 \\
\hline
\end{tabular}

El conocimiento de los pacientes sobre su diagnóstico y tratamiento actual para ansiedade (Tabla 3) varió siendo más frecuente en Montevideo - Uruguay 59s8\%, seguido de Brasilia
- Brasil $48.5 \%$ y Valparaíso - Chile, $44.9 \%$. Tales porcentajes contrastaron con Jamaica y Panamá, en donde correspondían a solamente $5.4 \%$ y $6.0 \%$ de los pacientes.

Tabla 3 - Tratamientos actuales realizados por profesionales de salud para ansiedad y depresión en los pacientes que buscaron atención en los centros de tratamiento para abuso y dependencia de drogas según ciudades y países, 2009/2010

\begin{tabular}{|c|c|c|c|c|c|c|c|c|c|c|c|c|c|c|c|c|}
\hline \multirow{3}{*}{ Ciudades / Países } & \multicolumn{8}{|c|}{ Ansiedad } & \multicolumn{8}{|c|}{ Depresión } \\
\hline & \multicolumn{2}{|c|}{$\mathbf{S i}$} & \multicolumn{2}{|r|}{ No } & \multicolumn{2}{|r|}{ NS $^{*}$} & \multicolumn{2}{|r|}{$\mathbf{N} \mathbf{R}^{\dagger}$} & \multicolumn{3}{|c|}{ Si } & \multicolumn{2}{|c|}{ No } & \multicolumn{2}{|c|}{ NS* } & \multirow{2}{*}{$\begin{array}{r}\mathbf{N R}^{\dagger} \\
\%\end{array}$} \\
\hline & $\mathrm{n}$ & $\%^{\ddagger}$ & $\mathbf{n}$ & $\%$ & $\mathbf{n}$ & $\%$ & $\mathbf{n}$ & $\%$ & $\mathbf{n}$ & $\%$ & $\mathbf{n}$ & $\%$ & $\mathbf{n}$ & $\%$ & $\mathrm{n}$ & \\
\hline Brasília/Brasil & 80 & 48.5 & 81 & 49.1 & 3 & 1.8 & 1 & 0.6 & 70 & 42.4 & 90 & 54.5 & 3 & 1.8 & 2 & 1.2 \\
\hline Macaé/Brasil & 23 & 38.3 & 37 & 61.7 & - & - & - & - & 17 & 28.3 & 43 & 71.7 & - & - & - & - \\
\hline Valparaíso/Chile & 48 & 44.9 & 41 & 38.3 & - & - & 18 & 6.8 & 30 & 28.0 & 50 & 46.7 & - & - & 27 & 25.2 \\
\hline $\begin{array}{l}\text { Ciudad Guatemala/ } \\
\text { Guatemala }\end{array}$ & 32 & 35.1 & 50 & 55.0 & 2 & 2.2 & 7 & 7.7 & 16 & 17.3 & 61 & 67.2 & 3 & 3.3 & 11 & 12.2 \\
\hline Parroquias/Jamaica & 3 & 5.4 & 53 & 94.6 & - & - & - & - & 11 & 19.6 & 45 & 80.4 & - & - & - & - \\
\hline León/Nicaragua & 7 & 17.1 & 30 & 73.2 & - & - & 4 & 9.8 & 6 & 14.6 & 27 & 65.9 & - & - & 8 & 19.5 \\
\hline D. Managua /Nicaragua & 39 & 30.0 & 91 & 70.0 & - & - & - & - & 33 & 25.4 & 97 & 74.6 & - & - & - & - \\
\hline Provincias/Panamá & 10 & 6.0 & 145 & 86.8 & 4 & 2.4 & 8 & 4.8 & 11 & 6.6 & 44 & 86.2 & 4 & 2.4 & 8 & 4.8 \\
\hline Asunción/Paraguay & 36 & 24.2 & 86 & 57.7 & 27 & 18.1 & 0 & - & 26 & 17.4 & 92 & 61.7 & 31 & 20.8 & 0 & - \\
\hline Montevideo/Uruguay & 79 & 59.8 & 46 & 34.8 & 6 & 4.5 & 1 & 0.8 & 44 & 33.8 & 72 & 55.4 & 10 & 7.7 & 4 & 3.1 \\
\hline
\end{tabular}

${ }^{*} \mathrm{NS}=$ no sabe; $\nmid \mathrm{NR}=$ no responde; $\ddagger$ Porcentajes pueden no sumar $100 \%$ debido a valores faltantes. 
De modo semejante, el diagnóstico y tratamiento actual de depresión fue más frecuente en Brasilia - Brasil, $42.4 \%$, seguido de Montevideo - Uruguay, 33.8\%, Macaé - Brasil y Valparaíso Chile, $\sim 28.0 \%$. Estos porcentajes contrastan con los alcanzados por Panamá, $6.6 \%$ y León - Nicaragua, $14.6 \%$. La proporción que no sabía acerca del diagnóstico actual de esos dos trastornos fue mayor en Asunción - Paraguay (aproximándose a 20\%). En los otros lugares tales porcentajes eran menores de 5,0\% para ansiedad y de $8.0 \%$ para depresión.

La tabla 4 resume los resultados de los cálculos de puntuaciones referentes a la escala de Kessler K-10, sobre la presencia de distrés psicológico. Los totales de pacientes en la columna de la izquierda corresponden al número de individuos en que todas las preguntas referentes a los 10 componentes de la escala K-10 fueron respondidas. Hubo variaciones importantes en la pre- valencia de distrés muy severo, mostrando que los mayores valores fueron alcanzados en León - Nicaragua, 55.7\%, aunque haya sido calculado con base en sólo 36 pacientes que respondieron todas las preguntas de la escala, seguido de la Ciudad de Guatemala, 43.1\% ; $(n=81)$, Montevideo - Uruguay, $42.2 \%(n=128)$, Brasilia - Brasil, 39\% $(n=164)$ y Macaé - Brasil, 34.0\% ( $n=58)$. El menor valor fue alcanzado por Asunción - Paraguay, $9.4 \%(\mathrm{n}=149)$.

El análisis conjunto de las categorías de distrés "severo" y "muy severo" (equivalente a las descritas por otros autores como "alto" y "muy alto"), mostró variaciones semejantes. Los mayores valores fueron alcanzados en León- Nicaragua, $80.7 \%$ ( $\mathrm{n}=36)$, seguido de Montevideo - Uruguay, $75.8 \%$, Ciudad de Guatemala, $71.5 \%$, Brasilia - Brasil, $70.1 \%$ y Macaé - Brasil, $61 \%$. El menor valor fue alcanzado por Asunción - Paraguay (26.7\%).

Tabla 4 - Grados de distrés psicológico en los últimos 30 días, referidos por los pacientes atendidos en los centros de tratamiento para abuso y dependencia de drogas según ciudades y países, 2009/2010

\begin{tabular}{|c|c|c|c|c|c|c|c|c|}
\hline \multirow{3}{*}{ Ciudades/Países } & \multicolumn{8}{|c|}{ Grado de distrés psicológico según escala K-10 (Kessler) } \\
\hline & \multicolumn{2}{|c|}{$\begin{array}{c}\text { Bajo } \\
(10-15)\end{array}$} & \multicolumn{2}{|c|}{$\begin{array}{c}\text { Moderado } \\
(16-21)\end{array}$} & \multicolumn{2}{|c|}{$\begin{array}{l}\text { Severo } \\
(22-29)\end{array}$} & \multicolumn{2}{|c|}{$\begin{array}{c}\text { Muy severo } \\
(30-50)\end{array}$} \\
\hline & $\mathrm{n}$ & $\%$ & $\mathrm{n}$ & $\%$ & $\mathbf{n}$ & $\%$ & $\mathbf{n}$ & $\%$ \\
\hline $\begin{array}{l}\text { Brasília/Brasil } \\
\mathrm{n}=164\end{array}$ & 31 & 19.0 & 18 & 10.9 & 51 & 31.1 & 64 & 39.0 \\
\hline $\begin{array}{l}\text { Macaé/Brasil } \\
\mathrm{n}=58\end{array}$ & 10 & 17.0 & 13 & 22.0 & 16 & 27.0 & 20 & 34.0 \\
\hline $\begin{array}{l}\text { Valparaíso/Chile } \\
\mathrm{n}=107\end{array}$ & 22 & 20.6 & 33 & 30.8 & 26 & 24.3 & 26 & 24.3 \\
\hline $\begin{array}{l}\text { Guatemala } \\
n=81\end{array}$ & 7 & 8.6 & 16 & 19.7 & 23 & 28.4 & 35 & 43.1 \\
\hline $\begin{array}{l}\text { Parroquias/Jamaica } \\
n=56\end{array}$ & 16 & 28.6 & 16 & 28.6 & 17 & 30.3 & 7 & 12.5 \\
\hline $\begin{array}{l}\text { León/Nicaragua } \\
n=36\end{array}$ & 2 & 5.5 & 5 & 13.8 & 9 & 25.0 & 20 & 55.7 \\
\hline $\begin{array}{l}\text { D. Managua/Nicaragua } \\
n=130\end{array}$ & 56 & 43.0 & 30 & 23.1 & 28 & 21.5 & 16 & 12.4 \\
\hline $\begin{array}{l}\text { Provincias/Panamá } \\
n=167\end{array}$ & 57 & 34.1 & 47 & 28.1 & 31 & 18.6 & 32 & 19.2 \\
\hline $\begin{array}{l}\text { Asunción/Paraguay } \\
n=149\end{array}$ & 73 & 49.0 & 36 & 24.3 & 26 & 17.3 & 14 & 9.4 \\
\hline $\begin{array}{l}\text { Montevideo Uruguay } \\
n=128\end{array}$ & 8 & 6.3 & 23 & 17.9 & 43 & 33.6 & 54 & 42.2 \\
\hline
\end{tabular}

La tabla 5 reúne los resultados de los cálculos de puntuaciones de la escala de APGAR familiar, sobre la presencia de disfuncionalidad familiar percibida por los pacientes. Los totales de pacientes en la columna de la izquierda corresponden al número de individuos que respondieron todas las preguntas referentes a los cinco componentes de la escala.
Hubo variaciones en la prevalencia de percepción de la familia como "severamente disfuncional". La mayor proporción fue alcanzada por pacientes tratados en centros de las provincias de Panamá, donde $34.7 \%$ de los 167 participantes percibían su familia de ese modo, seguido de León - Nicaragua y Departamento de Managua - Nicaragua (25.7\%) 
$(\mathrm{n}=39)$ y $23.1 \%(\mathrm{n}=130)$, respectivamente. En Montevideo - Uruguay, esa proporción fue cercana a $20 \%(n=130)$. En las Parroquias de Jamaica $(n=56)$ y Ciudad de Guatemala ( $\mathrm{n}=90)$, los valores fueron de $17,8 \%$, en las ciudades brasileñas, alrededor de $15 \%(n=151$ e $n=58)$. Los menores valores fueron alcanzados en Asunción - Paraguay (n=149) y en Valparaíso - Chile $(\mathrm{n}=107)$, con porcentajes de $8.5 \%$ y $11.2 \%$, respectivamente.
El análisis de la unión de las categorías "severamente disfuncional" y "moderadamente disfuncional" mostró valores más homogéneos siendo que la mayor proporción de esa suma de las dos categorías fue alcanzada por pacientes tratados en centros de Panamá (50.9\%), seguido de los sitios de estudio nicaragüenses y Montevideo - Uruguay, entre $45 \%$ y $47 \%$.

Tabla 5 - Grados de funcionalidad familiar percibida por los pacientes atendidos en los centros de tratamiento para abuso y dependencia de drogas según ciudades y países, 2009/2010

\begin{tabular}{|c|c|c|c|c|c|c|}
\hline \multirow{3}{*}{ Ciudades / Países } & \multicolumn{6}{|c|}{ Grado de funcionalidad familiar percibida según escala APGAR familiar(Smilkstein) } \\
\hline & \multicolumn{2}{|c|}{ Normofuncional (>7) } & \multicolumn{2}{|c|}{$\begin{array}{l}\text { Moderadamente } \\
\text { disfuncional (4-6) }\end{array}$} & \multicolumn{2}{|c|}{$\begin{array}{c}\text { Severamente } \\
\text { disfuncional }(<3)\end{array}$} \\
\hline & $\mathbf{n}$ & $\%$ & n & $\%$ & $\mathbf{n}$ & $\%$ \\
\hline $\begin{array}{l}\text { Brasília/Brasil } \\
n=161\end{array}$ & 96 & 59.6 & 41 & 25.4 & 24 & 15.0 \\
\hline $\begin{array}{l}\text { Macaé/Brasil } \\
\mathrm{n}=58\end{array}$ & 38 & 65.5 & 11 & 19.0 & 9 & 15.5 \\
\hline $\begin{array}{l}\text { Valparaíso/Chile } \\
\mathrm{n}=107\end{array}$ & 71 & 66.4 & 24 & 22.4 & 12 & 11.2 \\
\hline $\begin{array}{l}\text { Guatemala } \\
\mathrm{n}=90\end{array}$ & 53 & 58.8 & 21 & 23.4 & 16 & 17.8 \\
\hline $\begin{array}{l}\text { Parroquias/Jamaica } \\
\mathrm{n}=56\end{array}$ & 37 & 66.1 & 9 & 16.1 & 10 & 17.9 \\
\hline $\begin{array}{l}\text { León/Nicaragua } \\
\mathrm{n}=39\end{array}$ & 21 & 53.8 & 8 & 20.5 & 10 & 25.7 \\
\hline $\begin{array}{l}\text { D. Managua/Nicaragua } \\
n=130\end{array}$ & 71 & 54.6 & 29 & 22.3 & 30 & 23.1 \\
\hline $\begin{array}{l}\text { Provincias/Panamá } \\
\mathrm{n}=167\end{array}$ & 85 & 50.9 & 24 & 16.2 & 58 & 34.7 \\
\hline $\begin{array}{l}\text { Asunción/Paraguay } \\
n=149\end{array}$ & 104 & 70.0 & 32 & 21.5 & 13 & 8.5 \\
\hline $\begin{array}{l}\text { Montevideo/Uruguay } \\
n=131\end{array}$ & 71 & 54.2 & 34 & 26.0 & 26 & 19.8 \\
\hline
\end{tabular}

\section{DISCUSIÓN}

Con respecto a las características de los pacientes, en todos los centros estudiados hubo una mayoría de participantes de sexo masculino, lo que concuerda con la mayoría de los estudios latinoamericanos..$^{10}$ Otras características fueron diferentes de acuerdo con las ciudades y centros de tratamiento. Una explicación a los hallazgos discrepantes se fundamenta inicialmente en el tratamiento ofrecido por los respectivos centros y en la propia substancia que motivó el tratamiento. Un ejemplo es la distribución encontrada por edad. Algunos centros presentaron personas muy jóvenes (Montevideo), en donde la sustancia más importante era la pasta base de coca, contrastando con otros en que la media de edad fue más elevada (Brasilia) y donde predominaba la dependencia de alcohol. Otras drogas se mostraron predominantes en otros lugares. La cocaína en polvo casi no fue mencionada en Managua, mientras que en Panamá y Valparaíso, donde los tamaños de población son comparables, una cuarta parte de los pacientes la nombraron.

La prevalencia de comorbilidades referida por los pacientes puede ser asimilada a las respuestas dadas por participantes de encuestas epidemiológicas realizadas en la base de la población, preguntando por la presencia de la enfermedad en los últimos 30 días o en los últimos 12 meses. En este sentido, resulta claro que las prevalencias observadas en el presente estudio son mayores que las obtenidas en la población. ${ }^{11}$

En el presente estudio la prevalencia de distrés psicológico, medido por la escala K-10, difería entre los diferentes lugares. En centros con 
tamaños de muestra de más de 100 pacientes, fue más alta en centros del Uruguay (Montevideo), Brasil (Brasilia) y Chile (Valparaíso), siendo en estos centros mayor de $48 \%$. En las provincias de Panamá y Departamento de Managua - Nicaragua, los valores fueron intermediarios entre 30\% y $40 \%$ y en Asunción - Paraguay, más bajos ( 27\%). En estudio realizado en Australia, ${ }^{12}$ los autores encontraron niveles de distrés severo y muy severo en $30 \%$ entre jóvenes australianos en custodia aplicando la misma escala. En investigación semejante, con jóvenes que habían cometido delitos en el mismo país, ese porcentaje fue $25 \%$. Cuando utilizaban más de una droga, este porcentaje subía a $38 \%{ }^{13}$

Un grupo de investigación ${ }^{14}$ ha estudiado la validación de instrumentos de screening para trastornos mentales en personas con abuso/ dependencia. Los autores utilizaron el mismo instrumento en poblaciones diferentes concluyendo que no puede darse por consensuado que la validad sea la misma en distintos ámbitos y poblaciones. En el presente estudio, los pacientes de América Central estaban siendo tratados en su mayoría en instituciones de tipo ONG religiosas en que muchas veces la presencia de profesionales era mínima. Esto contrasta con otras poblaciones de estudio en que las instituciones eran públicas (Brasilia y Montevideo; en Valparaíso y Macaé eran de más de un tipo). Es probable que la visión o percepción de los síntomas de ansiedad y depresión puedan diferir dependiendo de la filosofía subyacente o del enfoque terapéutico. Esto afectaría las respuestas al K-10.

Es concebible que las modalidades de tratamiento, sus principios y presupuestos atraviesen la percepción de los pacientes sobre su vivencia frente al problema. El proceso de recuperación implica también en un estrés adicional resultante de intentos de mantenerse en adhesión y de cumplir expectativas. El estrés puede estar asociado a abstinencia. ${ }^{15}$

Con respecto a la percepción de la funcionalidad familiar, medida a través del APGAR familiar, los resultados fueron sorprendentes en el sentido de que había porcentajes relativamente bajos de disfuncionalidad, la mayoría entre $10 \%$ y $20 \%$. Las excepciones fueron los valores alcanzados en Panamá $(\sim 35 \%)$ y Managua-Nicaragua ( 23\%), entre los locales con mayor tamaño de muestra. En Panamá, fue en donde se observaron las mayores proporciones de pacientes viviendo solos o en la calle. En general, se acepta que altos niveles de disfunción familiar en las áreas de capacidad de respuesta afectiva y desempeño de roles están asociados con mayores frecuencias de abuso de substancias. ${ }^{6,16}$

\section{CONCLUSIONES}

Con respecto a la atención que se les brinda a los pacientes participantes del estudio en los centros de tratamiento por abuso/dependencia de drogas, podemos concluir que apenas una proporción de $30 \%$ a $40 \%$ de los pacientes tienen un diagnóstico psiquiátrico. Podemos concluir que la prevalencia del distrés psicológico en los pacientes de los lugares con mayores tamaños de muestra indicando tiende a ser mayor de $30.0 \%$, reflejando probablemente la mayor prevalencia de depresión y ansiedad. Los niveles de funcionalidad familiar no son tan altos como podría esperarse y se situaron por debajo de $20.0 \%$ en la mayor parte de los centros estudiados.

\section{Limitaciones}

Entre las limitaciones previstas inicialmente en esta investigación están las relacionadas con el diseño del estudio transversal que son reconocidas en la literatura epidemiológica. ${ }^{17}$ Otras surgen de las características de los centros de tratamiento, que dependen de la estructura sanitaria existente y la organización de los servicios de salud. Por lo tanto, hay certeza que el sesgo de referencia institucional y de diferentes situaciones de acceso a los servicios de salud, limitan la capacidad de generalizar los resultados.

El presente estudio también puede ser limitado debido al uso de un cuestionario autoadministrado como la principal fuente de recopilación de datos. Se trató de minimizar problemas con la ayuda proporcionada por los asistentes de investigación capacitados o el chequeo de los cuestionarios en el momento de la entrega. En lo tocante a los antecedentes de consumo de sustancias y al conocimiento sobre diagnósticos previos y actuales de enfermedades mentales, lo referido por los pacientes no fue validado.

Otra posible limitación es la propia escala para medir el distrés psicológico. Es posible que diferencias culturales hagan más difícil aceptar estados de depresión y ansiedad, dimensiones para las cuales la escala es más sensible, además del estigma asociado a trastorno mental. Esto podría ser particularmente difícil entre hombres, que formaron la mayor parte de las poblaciones de pacientes. Por la mayor sensibilidad para depresión y ansiedad, otros trastornos no serían detectados. 
Finalmente, los datos hasta aquí analizados reportan estimativas no ponderadas de prevalencias que ciertamente sufren el efecto de tamaños de muestra muy diferentes. Tales números eran pequeños en algunos lugares (Jamaica=29 individuos; León-Nicaragua $=41$; Macaé-Rio de Janeiro=60), por lo cual las prevalencias obtenidas deben ser analizadas con cautela.

\section{Recomendaciones}

Con base en los resultados observados, podemos recomendar la introducción en la curricula de las carreras de pregrado y postgrado de las profesiones de salud (médicos, enfermeros, psicólogos), de actividades de capacitación sobre la importancia de la comorbilidad psiquiátrica y de su detección en el ámbito del tratamiento para abuso/dependencia de drogas.

En los centros de tratamiento especializado es necesario realizar un diagnóstico operacional para evaluar su capacidad de manejo de pacientes con comorbilidad, los factores facilitadores y dificultadores, las necesidades y factibilidades de su superación, la articulación con niveles diferentes del sistema de salud. También puede ser necesario, dependiendo del escenario institucional, sensibilizar a los profesionales que trabajan en esos centros sobre la existencia y relevancia de la comorbilidad.

Dependiendo de la capacidad de los centros, puede ser necesaria la implementación del rastreo (screening) para detección de comobilidades. ${ }^{18-19}$ Para eso sería necesario determinar atribuciones: quién lo realiza, con qué herramienta o instrumento, quién hace la evaluación diagnóstica ulterior. Tal implantación supone también la capacitación de los profesionales.

Esto es necesario para la posterior organización de la cobertura en cuidados para los trastornos más frecuentes (probablemente, ansiedad y depresión), en el propio centro de tratamiento especializado, en lo posible sin referencia a otras instancias de salud.

Cuando los trastornos extrapolen la capacidad de resolución del centro de tratamiento para alcohol y drogas, debe ser garantizada la referencia para otros profesionales o ámbitos del sistema de salud.

\section{AGRADECIMIENTOS}

Este estudio se realizó gracias al apoyo, asesoría y patrocinio del Gobierno de Canadá; de la
Organización de los Estados Americanos (OEA); de la Secretaria de Seguridad Multidimensional (SSM); de la Comisión Interamericana para el Control del Abuso de Drogas (CICAD); y del Centro de Adicciones y Salud Mental (CAMH - Ontario/ Canadá). También se agradece la colaboración de otros colegas que contribuyeron, de forma directa o indirecta, a la implementación del estudio y finalmente, a los pacientes que participaron.

\section{REFERENCIAS}

1. Regier D, Farmer M, Rae D, Locke B, Keith S, Judd L, Goodwin F. Co-morbidity of mental disorders with alcohol and other drug abuse: results from the epidemiologic catchment area (ECA) study. JAMA. 1990 Nov 21; 264(19):2511-8.

2. Evans K, Sullivan JM. Dual diagnosis. counseling the mentally III substance abuser. United States of America: The Guilford Press; 2001.

3. Dalex DC, Moss H B. Dual disorders. counseling clients with chemical dependency and mental illness. $3^{\mathrm{a}}$ ed. Minnesota (US): Hazelden Foundation Center, 2002.

4. Brady KT. Comorbidity with substance abuse [editorial]. Medscape [online]. 2003 [access 2009 Jun 24]. Available at: http://cme.medscape.com/ viewarticle/457178

5. Kessler RC, Andrews G, Colpe LJ, Hiripi E, Mroczek DK, Normand SLT, et al. Short screening scales to monitor population prevalances and trends in nonspecific psychological distress. Psychol Med. 2002 Aug; 32(6):959-76.

6. McKay JR, Murphy RT, Rivinus TR, Maisto, SA. Family dysfunctions and alcohol and drug use in adolescent psychiatric patients. J Am Acad Child Adolesc Psychiatry. 1991 Nov; 30(6):967-72.

7. Smilkstein G, Ashworth C, Montano D. Validity and reliability of the Family APGAR as a test of family function. J Fam Prac. 1982 Aug; 15(2):303-11.

8. Silveira J. Co-morbidade psiquiátrica em dependentes de substâncias psicoativas: resultados preliminares. Rev Bras Psiqui. 1999 Jul-Set; 21(3):145-51.

9. Andrews G, Slade T. Interpreting scores on the Kessler psychological distress scale (K10). Aust N Z J Public Health. 2001 Dec; 25(6):494-7.

10. Saraceno B, Saxena S, Caraveo-Adnurga J, Khon R, Levav I, Caldas de Almeida M. Los trastornos mentales en América Latina y el Caribe: asunto prioritario para la Salud Publica. Rev Panam Salud Pública. 2005 Nov; 18(4-5):229-40.

11. Mari JJ, Jorge MR, Kohn R. Epidemiologia dos transtornos psiquiátricos em adultos. In: Mello MF, Mello AAF, Kohn R, organizadores. Epidemiologia da saúde mental no Brasil. Porto Alegre (RS): Artmed; 2007. p.119-41. 
12. Allerton M, Champion U, Beilby R, Butler T, Fasher M, Kenny D, et al. NSW young people in custody health survey: key findings report. Sydney (AU): NSW Department of Juvenile Justice; 2003.

13. Kenny DT, Nelson PK. Young offenders on community orders: health, welfare and criminogenic needs. Sydney (AU): Sydney University Press; 2008.

14. Castel S, Rush B, Scalco M. Screening of mental disorders among clients with addictions. The need for population-specific validation. Int J MentHealth Addiction. 2008 Jan; 6(1):64-71.

15. Miele GM, Trautman KD, Hasin DS. Assessing comorbid mental and substance-use disorders: a guide for clinical practice. J Prac Psych Behav Health. 1999 Sep; 5:272-82.

16. Sousa M. Perfil dos utilizadores de psicofármacos na
Unidade de Saúde de Canelas. Rev Port Clín Geral. 2007; 23:33-42.

17. Newman TB, Browner WS, Cummings SR, Hulley $\mathrm{SB}$. Designing cross-sectional and case control studies. In: Hulley SB, Cummings SR, Browner WS, Grady DG, Newman TB, editors. Designing clinical research: an epidemiological approach. $3^{\text {rd }}$ Ed, Philadelphia (US): Lippincott, Williams and Wilkins, 2001. p. 127-44.

18. Rush B. Editorial. Int J Ment Health Addiction. 2008 Jan; 6(1):1-6.

19. Zimmerman, M. To screen or not to screen: conceptual issues in screening for psychiatric disorders in psychiatric patients with a focus on the performance of the psychiatric diagnostic screening. Int J Ment Health Addiction. 2008 Jan; 6(1):53-63. 